\title{
Risks of minimally invasive surgery underestimated: a report of the Dutch Health Care Inspectorate
}

\author{
Laurents P. S. Stassen · Willem A. Bemelman • \\ Jeroen Meijerink
}

Published online: 22 July 2009

(c) The Author(s) 2009. This article is published with open access at Springerlink.com

Endoscopic surgery is known for its different eye-hand coordination and its learning curves. It encompasses complex and sensitive equipment that requires different maintenance and quality control. Despite the development of dedicated training programs and extensive research justifying the endoscopic revolution, the Dutch Health Care Inspectorate (DHI) [1] recently surprised endoscopic surgeons with its report entitled Risks of Minimally Invasive Surgery Underestimated [2]. In this report, the Inspectorate expressed its sincere concern regarding endoscopic surgery in The Netherlands.

The DHI report was based on investigations done by the Inspectorate initiated by unusual observations of incidents associated with endoscopic surgery. The investigations consisted of questionnaires and on-site visits to hospitals and endoscopic surgeons. Such activity is part of DHI's task to control the quality of health care in general.

Is this an example of the changing attitude of authorities and the public toward advances in health care, or did we really fail in doing our homework properly? The first question can be answered in the affirmative. Safety in

\footnotetext{
L. P. S. Stassen ( $\square)$

Department of Surgery, Maastricht University Medical Center, P.O. Box 5800, 6202 AZ Maastricht, The Netherlands

e-mail: 1stassen@xs4all.nl
}

W. A. Bemelman

Department of Surgery, Academic Medical Center, P.O. Box

22700, 1100 DD Amsterdam, The Netherlands

e-mail: w.a.bemelman@amc.uva.nl

J. Meijerink

Department of Surgery, Free University Medical Center,

Amsterdam, The Netherlands

e-mail: j.meijerink@vumc.nl health care is high on the agenda. In 2004, the Dutch Shell CEO Rein Willems, invited by the Minister of Healthcare, published a report on the appropriately entitled topic You Work Here Safely, or You Don't Work Here. To answer the second question, we have to look into the risks observed and the actions taken to reduce these risks.

The observed incidents were miscellaneous, some going back as far as the introduction of endoscopic surgery (e.g., common bile duct injury in laparoscopic cholecystectomy, ureteric injury during colonic resection, visceral vascular injury during nephrectomy, and injury due to defects and misuse of instruments and apparatus). The DHI concluded that the actions taken to prevent such incidents were insufficient. The required minimal dexterity was not broadly defined. Training in endoscopic surgery needed to be improved and to be more uniform between specialties. A system for structured assessment of the performer's skills was lacking. Registration of endoscopic procedures and its complications was insufficient. Uniformity among the different users in the same hospital often was lacking in terms of technique, training, and apparatus used. Quality control of apparatus and endoscopic surgery in general was insufficient.

It might be argued that the observations were not based on prospective nationwide databases or registries and therefore of limited value. It also might be argued that although endoscopic research disclosed phenomena such as the learning curves and the fallibility of man-machine interaction, this does not mean that these phenomena must be exclusively attributed to this type of surgery. Because of such awareness and the continuous effort of endoscopic surgeons to improve safety and the standard of care, the criticism was felt to be unexpectedly strong.

Yet, the incidents cannot be denied. What efforts did we make to avoid these? 
Dedicated training was developed early and has been incorporated into residency. Junior residents follow the compulsory Basic Laparoscopy Course. Seniors take advanced courses at Elancourt and Strasbourg, organized by Dutch faculty. Many workshops and nationwide courses are offered to registered surgeons. Best practices of endoscopic techniques have been described. Solid research has been conducted to establish the value and hazards of endoscopic procedures such as inguinal hernia repair [3], resection of colonic cancer [4] and Nissen fundoplication [5]. Other research has focused on hand-eye coordination and improvement of training and safety in logistics and apparatus [6].

Endoscopic surgery has been professionally organized. The Dutch Associations of Surgery, Gynecology, and Urology each have their working groups on endoscopic surgery, and these groups are federally united in the Dutch Association of Endoscopic Surgery.

Probably, we could have done better. Discrepancies in performance between surgeons and between institutes may have been tolerated too long. A certain learning curve was accepted because it was considered short compared with the revolution that took place. Although the learning curve and time frame indeed were much shorter than for previous changes in health care, the tolerance of the community for such imperfections had changed at the same time.

Also, authorities could have done better. For a long time, no registration codes existed for endoscopic procedures. This prohibited proper administration of procedures and their complications and proper reimbursements for endoscopic surgery. Such a lack of facilitation hampers progress.

What measures are demanded by the DHI? At the national level, DHI is requiring establishment of pan-disciplinary agreements with regard to training and skills assessment in endoscopic surgery, the introduction of a quality assurance method (e.g., certification) covering endoscopic skills, the introduction of guidelines and protocols for the inspection and maintenance of endoscopic instruments and equipment, and the development of a model of a quality system for endoscopic surgery.

At the hospital level, the measures should include a description of hospital policy regarding endoscopic surgery, establishment of a quality system for endoscopic procedures based on national guidelines, implementation of adequate registration facilities for endoscopic surgery and assessment of their usage, establishment of pan-disciplinary user consultation to facilitate hospital policy and to evaluate outcome, publication of an annual report on endoscopic surgery, and evaluation and peer review of the hospital staff's endoscopic skills based on national criteria.

The Dutch endoscopists chose to regard the criticism as valuable advice and aimed at implementing most measures.
Not all demands could be met. For some demands, evidence to guide proper action was lacking.

A multidisciplinary approach was taken. A plan of action was devised in cooperation with all working groups, their specialist organizations, and the Dutch Association of Endoscopic Surgery. The plan of action was meant to serve as a blueprint for each hospital's individual plan of action. Where appropriate, information from the European Association for Endoscopic Surgery 2006 Guidelines for Endoscopic Surgery was used.

At the national level, "guidelines and protocols for the inspection and maintenance of endoscopic instruments and equipment" were formulated and "a model for a quality system for endoscopic surgery" was proposed. Best practices were added to those already existing, then proposed to the appropriate national bodies for approval.

By formulating the measures of "agreements with regard to training and skills assessment" and the "introduction of a quality assurance method (e.g., certification) covering endoscopic skills," the DHI more specifically meant defining exact required numbers of procedures performed for certification and development of a system for video-based competency analysis.

These demands are difficult to meet. Exact numbers of procedures are hard to give. Learning curves are identified and appreciated. Such is the case with laparoscopic colonic surgery for cancer, which resulted in the Society of American Gastrointestinal Endoscopic Surgeons (SAGES)/ American Society of Colon and Rectal Surgeons 2004 statement that "prerequisite experience should include at least 20 laparoscopic colorectal resections with anastomosis for benign disease or metastatic colon cancer before using the technique to treat curable cancer." Yet, this statement must be seen as a starting point and the number of procedures as a minimum requirement.

On the other hand, reports on endoscopic inguinal hernia repair illustrate the relativity of a learning curve. The number of procedures required before proficiency is reached varies from 30 to $250[3,7]$. An exact figure gives a false impression of competence or safety. Even continuous practice does not make perfect. Lawyers and managers are inclined to use such numbers as objective quality parameters, which they are not. Outcome is what matters. Therefore, there is a great reluctancy to mention numbers indicating proficiency. Only internationally recognized figures defining the minimum required level of experience are used.

Video-based analysis is another controversial issue. It may seem to be an ideal method for determining dexterity, especially considering the broad availability of endoscopic images. However, assessment methods should be feasible, reliable, and valid for the circumstances under which they are used. Video-based analysis has been proved valid for 
differentiating between novices and experts at the level of generic motor skills [8]. This is not yet the case for assessing the detailed performance of procedures, nor for monitoring the development of skills during surgical training or for establishing a difference in dexterity between endoscopic surgeons. Use of insufficiently designed methods results in conflicting data, as observed in the evaluation of surgical technique [9].

Premature introduction carries another important risk. Third parties not aware of the method's insufficient design may use it for litigation issues. Available images can be claimed and offered to experts for assessment. The weakness of expert opinion in the absence of a valid assessment method has been shown in common bile duct injury malpractice litigation [10]. Poor interobserver reliability was found when operative management and technique were evaluated by expert witnesses without prior consensus on the essential steps and without a validated assessment method. Video-based analysis needs thorough further development before it can be used to assess the proficiency of endoscopic surgeons.

Some of the measures at the hospital level were relatively easy to implement. Based on available knowledge, existing local initiatives, and informal interdisciplinary consultation, clear guidelines have been formulated for the safe use and maintenance of apparatus, for the introduction of new apparatus, for instruments and technology, and for the setting up of multidisciplinary management teams.

The claim for proper registration of procedures and complications was addressed earlier in this report. Its necessity seems obvious. However, until 2006, Dutch national authorities had only sparingly issued registration codes for endoscopic procedures despite requests of the surgical society. In this respect, the current claim of the DHI is welcome. A wide variety of codes has been introduced in the meantime, enabling registration of endoscopic procedures and their complications.

The recent attention on endoscopic surgery has had another welcome side effect. The measures that must be taken require personnel and funds. In a financial health care system characterized by continuous constraints, the DHI report will help in directing resources toward endoscopic surgery.

The criticism received leads to the question whether the attitude of authorities and the public had changed or whether we failed in doing our homework properly. It is evident that the attitude of the community toward innovation has changed. Paraphrasing on the aforementioned safety report entitled You Work Here Safely or You Don't Work Here, the current attitude might be characterized as "You introduce safely or you don't introduce." Yet, the responsible professionals did not do a bad job in introducing endoscopic surgery. Existing standards were followed, with much effort put into the safe development of endoscopic surgery. But with the changes we introduced, the world around us changed as well. Whereas learning curves used to be accepted in the past as inevitable side effects of innovation, the tolerance of the community for this phenomenon has been declining rapidly.

New standards need to be set. Professionals are already doing this. The National Orifice Surgery Consortium for Assessment and Research/SAGES white paper on the introduction of natural orifice translumenal endoscopic surgery (NOTES) is a proper example. The white paper states that before clinical introduction of the technique, fundamental issues have to be understood, which can be best addressed in the laboratory setting, after which approval of regulatory agencies should be obtained. The focus is shifting from mere innovation to safety, training, and assessment. Professionals will have to take responsibility and stay in the lead of this process, but authorities are needed as partners. Clear guidelines concerning the logistics of endoscopic surgery are described. Phase 1 has been completed. The second phase, broad implementation of these guidelines, might be an even greater task.

Open Access This article is distributed under the terms of the Creative Commons Attribution Noncommercial License which permits any noncommercial use, distribution, and reproduction in any medium, provided the original author(s) and source are credited.

\section{References}

1. http://www.igz.nl/uk

2. http://www.igz.nl/15451/475693/2007-11_rapport_minimaal_ in1.pdf

3. Liem MS, van der Graaf Y, van Steensel CJ, Boelhouwer RU, Clevers G-J, Meijer WS, Stassen LPS, Vente JP, Weidema WF, Schrijvers AJP, van Vroonhoven ThJMV (1997) Comparison of conventional anterior surgery and laparoscopic surgery for inguinal hernia repair. New Engl J Med 336:1541-1547

4. Colon Cancer Laparoscopic or Open Resection Study Group, Buunen M, Veldkamp R, Hop WC, Kuhry E, Jeekel J, Haglind E, Påhlman L, Cuesta MA, Msika S, Morino M, Lacy A, Bonjer HJ (2009) Survival after laparoscopic surgery versus open surgery for colon cancer: long-term outcome of a randomized clinical trial. Lancet Oncol 10:44-52

5. Draaisma WA, Rijnhart-de Jong HG, Broeders IA, Smout AJ, Furnee EJ, Gooszen HG (2006) Five-year subjective and objective results of laparoscopic and conventional fundoplication: a randomized trial. Ann Surg 244:34-41

6. Verdaasdonk EEG, Stassen LPS, Hoffmann WF, van der Elst M, Dankelman J (2008) Can a structured checklist prevent problems with laparoscopic equipment? Surg Endosc 22:2238-2243

7. Neumayer L, Giobbie-Hurder A, Jonasson O, Fitzgibbons R, Dunlop D, Gibbs J (2004) Open mesh versus laparoscopic mesh repair of inguinal hernia. N Engl J Med 350:1819-1827

8. Aggarwal R, Grantcharow T, Moorthy K, Milland T, Darzi A (2008) Towards feasible, valid, and reliable video-based assessments of technical surgical skills in the operating room. Ann Surg 247:372-379 
9. Verdaasdonk EEG, Dankelman J, Lange JF, Stassen LPS (2008) Transfer validity of laparoscopic knot-tying training on a VR simulator to a realistic environment: a randomized controlled trial. Surg Endosc 22:1636-1642
10. De Reuver PR, Dijkgraaf MG, Gevers SK, Gouma DJ, BILE study group (2008) Poor agreement among expert witnesses in bile duct injury malpractice litigation: an expert panel survey. Ann Surg 248:815-820 